\title{
4) Le visa : instrument de la mise à distance des
}

\section{Elspeth Guild et Didier Bigo}

\section{(2) OpenEdition}

1 Journals

\section{Édition électronique}

URL : http://journals.openedition.org/conflits/933

DOI : $10.4000 /$ conflits.933

ISSN : $1777-5345$

Éditeur :

CCLS - Centre d'études sur les conflits lilberté et sécurité, L'Harmattan

\section{Édition imprimée}

Date de publication : 1 mars 2003

Pagination : 82-95

ISBN : 2-7475-4868-8

ISSN : 1157-996X

Référence électronique

Elspeth Guild et Didier Bigo, «4) Le visa : instrument de la mise à distance des », Cultures \& Conflits [En ligne], 49 | printemps 2003, mis en ligne le 29 septembre 2003, consulté le 30 mars 2021. URL : http:// journals.openedition.org/conflits/933; DOI : https://doi.org/10.4000/conflits.933

Ce document a été généré automatiquement le 30 mars 2021.

Creative Commons License 


\title{
4) Le visa : instrument de la mise à distance des
}

\author{
Elspeth Guild et Didier Bigo
}

4) Le visa : instrument de la mise à distance des « indésirables »

Le visa n'est pas simplement un instrument technique. Il n'est même pas uniquement une stratégie plus efficace de gestion des frontières. Il est centralement de l'ordre du Politique. C'est à travers le visa que l'on tient à distance et que l'on met à l'écart les étrangers indésirables. C'est aussi à travers lui que l'on définit entre les pays de l'espace Schengen une liste d'ennemis non déclarés mais dont on tient à se protéger. En ce sens, le visa Schengen, en particulier sa cartographie, est susceptible d'une lecture schmittienne par la désignation qui est faite de la limite entre les amis et les ennemis. Mais nous verrons que les gouvernements et la Commission préfèrent une politique plus ambiguë et cherchent à brouiller les frontières, tout comme ils ne tiennent nullement à déclarer ce qu'ils font. La stratégie de faible visibilité n'empêche néanmoins pas sur le fond l' importance politique du sujet et son impact sur les libertés publiques.

La détermination des listes des pays soumis ou non à visa

Le contenu du règlement et des instructions consulaires communes : les critères utilisés

L'article 62 , point 2 b) i confère, nous l'avons vu, une compétence exclusive à la Communauté pour arrêter la liste des pays tiers dont les ressortissants sont soumis à l'obligation de visa et de ceux dont les ressortissants sont exemptés de cette obligation. Mais quelles sont les relations entre la Commission et les Etats membres du Conseil ? Quels critères vont être utilisés? En pratique qu'est-ce que cela signifie ? Comment les autorités consulaires vont-elles agir dans les pays dont les ressortissants sont soumis à visa?

Si les luttes sont fortes entre les Etats et la Commission en ce qui concerne les procédures et leurs enjeux, il est moins évident de distinguer sur le fond entre une position de la Commission et une position du Conseil. La direction de la DG JAI a appuyé une politique musclée contre l' immigration clandestine ayant les mêmes tonalités que les politiques gouvernementales allemandes, françaises ou britanniques. En revanche d' 
autres acteurs ont essayé d'obliger les gouvernements à préciser leur position, à révéler les critères effectifs des choix et à montrer les dangers d'un racisme institutionnel ou d'une discrimination par l'argent qui s'exprimeraient dans les textes de l'instruction consulaire. Ceci a été relayé par certains gouvernements dont la Belgique et par des ONG. Mais le débat est resté feutré. Tout le monde a voulu tabler sur l'efficacité attendue des mesures et sur le fait que cela évitait la polémique sur les contrôles aux frontières. Ce qui a été en jeu a tenu avant tout à la discussion sur les critères retenus, en particulier les exigences concernant les financements pour couvrir les frais de voyage et de court séjour ainsi que les critères sur le risque migratoire.

Nous allons citer quelques passages du règlement et des instructions consulaires communes afin de montrer comment se déploie l'ordre discursif de ces textes. Afin d'expliquer quels pays se trouvent sur la liste, et pourquoi ils s'y trouvent, la Commission a publié un texte sur les "Critères qui ont été utilisés pour déterminer l'appartenance d'un pays tiers " à l'annexe $\mathrm{I}^{1}$ ou à l'annexe $\mathrm{II}^{2}$ :

Comme elle le dit "pour déterminer si les ressortissants d'un pays tiers sont soumis à l'obligation de visa ou si au contraire ils sont exemptés de visa, il convient de prendre en considération un ensemble de critères pouvant être regroupés sous trois rubriques principales :

- L'immigration illégale : le régime de visas est un instrument essentiel de maîtrise des flux migratoires. A cet effet, il y a lieu de se référer à un certain nombre d'informations ou indicateurs statistiques pertinents pour l' appréciation du risque de flux migratoires illégaux (par exemple les informations et /ou statistiques concernant les séjours irréguliers, les refus d'entrée sur le territoire, les mesures d'éloignement, les filières d' immigration clandestine ou de travail clandestin), d'évaluer la sécurité des documents de voyage délivrés par le pays tiers concerné et enfin de tenir compte de l'existence et du fonctionnement des accords de réadmission conclus par ce pays ;

- L'ordre public : les constatations faites en particulier dans le cadre de la coopération policière peuvent mettre en évidence les caractéristiques de certains types de criminalités. Selon le degré de gravité, de permanence et d'étendue territoriale des criminalités en cause, le recours à l'obligation de visa peut être un des moyens de réponse à envisager. Les menaces à l' ordre public peuvent revêtir dans certains cas une gravité telle qu'elles mettent en cause la sécurité intérieure même d'un ou de plusieurs Etats membres. Le recours à l'instrument de l'obligation de visa, assumé solidairement par les autres Etats membres, peut être une des réponses appropriées à mettre en ouvre $^{3}$.

- Les relations internationales : le choix du régime de visas retenu à l' égard d'un pays tiers peut être un des moyens de souligner la qualité des relations que l'Union entend établir ou maintenir avec ce pays. A cet égard, il s'agit rarement des relations de l'Union avec un pays isolé. Le plus souvent, on a affaire aux relations de l'Union avec des groupes particuliers de pays et le choix d'un régime de visa a également des implications en termes de cohérence régionale. Le choix d'un régime de visa peut aussi refléter au départ la position particulière d'un Etat membre vis-à-vis d'un pays tiers, position à laquelle les autres Etats membres se rallient comme expression de la solidarité des Etats membres. Le critère de la réciprocité, auxquels les Etats recouraient individuellement et séparément dans le cadre des relations traditionnelles du droit 
international public, doit être désormais utilisé en tenant compte des exigences des relations extérieures de l'Union européenne avec les pays tiers $»^{4}$.

Et la Commission ajoute plus loin: "Compte tenu de l'extrême diversité des situations qui caractérisent les pays tiers ainsi que des relations qu'ils entretiennent avec l'Union européenne et les Etats membres, les critères énumérés ne peuvent pas être appliqués de manière automatique, par le biais de coefficients fixés à l'avance. Ces critères doivent être considérés comme des instruments d'aide à la décision à mettre en ouvre de manière souple et pragmatique, en faisant jouer des pondérations appropriées au cas par cas ».

A la lecture de ces critères et de leur combinaison, on est alors conduit à rebours vers la question de savoir si le visa sanctionne un individu particulier ou est un instrument de relations internationales entre les Etats? Le franchissement de la frontière de l'espace Schengen par un individu est-il dépendant de l'appréciation de son pays comme menace ? Ou un pays devient-il menaçant parce que ses ressortissants veulent le fuir?

Comment se fait l'inscription d'un pays sur la liste des pays soumis à visa? N'y a t il pas inversion des critères traditionnels des relations internationales où l'Etat fait l'individu par celui où les individus font cet Etat? Plus exactement n'y a-t-il pas une dialectique négative qui s' instaure et permet de lier « risque » idéologique d'un Etat et « risque » migratoire des individus?

L'appréciation de l'inscription du pays sur la liste des pays dont les ressortissants sont soumis à visa obligatoire sanctionne semble-t-il des politiques gouvernementales selon un critère de relations internationales - mais aussi des pratiques sociales individuelles qui relèvent d'un groupe identifiable localement ou ethniquement - selon des critères d'ordre public et de migration - qui poussent à inscrire un pays dans son ensemble sur la liste des pays dont les ressortissants sont soumis à visa.

Or, si l'individu est ressortissant d'un pays considéré comme pays à risque d'après les critères de la Commission, il est soumis à un visa obligatoire, quelles que soient ses qualités personnelles. Bref vivre dans un pays où il existe des minorités considérées comme dangereuses par d'autres pays est quasiment l'équivalent d'un délit ${ }^{5}$. Il en va de même des pays à fort taux migratoire. Et dans ce dernier cas, comment peut-on juger à l'avance du risque d'illégalité et empêcher que des individus qui veulent simplement visiter de la famille ou des amis, ne soient soupçonnés d'être, comme certains de leurs compatriotes, des « volontaires à l'immigration clandestine »?

Ceci veut dire, comme nous l'avons signalé en introduction que la frontière pour les ressortissants sur la liste des pays à visa obligatoire se trouve à leur point de départ, dans les pays tiers. La première frontière pour ces gens est au consulat d'un pays Schengen dans leur propre pays. La décision cruciale en ce qui les concerne sera prise dans ce consulat, au sein de la coopération consulaire des quinze, qui devient à cette échelle locale une coopération qui est parfois à dix sept car, ici, Royaume-Uni et Irlande acceptent de temps en temps de participer localement sans se sentir liés par les décisions prises à Bruxelles. Cette délocalisation virtuelle de la frontière qui la place avant le voyage - et non pendant ou après - est centrale dans la stratégie des pays Schengen et surtout de la Commission. Elle vise à « tarir les flux à la source » comme le disait brutalement un de nos interlocuteurs.

Elle ne s'applique donc pas à tous les pays mais aux pays dits à risque - sans autre précision que les trois critères très généraux cités plus haut: les relations internationales, l'ordre public et l'immigration clandestine ${ }^{6}$. 
Cela a un avantage certain pour tous les pays qui échappent à la procédure du visa pour leurs ressortissants. Ceux-ci ne sont pas triés sur place et ont souvent des contrôles allégés aux frontières. Ils profitent du fait qu' on considère tous leur ressortissants comme des « amis » en puissance. La carte que nous avons établie montre comment ces 44 Etats se répartissent : elle ressemble très fortement à la carte des pays développés, aux pays qui vont entrer dans l'Union européenne et à une large partie des Amériques. Sans qu'elle soit totalement celle du peuplement blanc puisque le Japon et Singapour en font partie, elle s'en rapproche néanmoins.

En revanche la liste des 133 pays (Etats et entités territoriales) dont les ressortissants sont soumis à visa recouvre clairement l'Afrique, le Moyen-Orient (à l'exception d'Israël) et une large partie de l'Asie. Et il devient clair que les critères de danger en matière de sûreté de l'Etat, danger lié au terrorisme et au crime sont, sans pudeur particulière, associés au danger « migratoire».

Le processus de repérage de ces « dangers » se fait d'ailleurs en deux temps qui doivent être distingués : celui qui concerne les pays, celui qui concerne les individus.

Le premier est la décision de mettre certains pays au sein de la liste soumise à visa obligatoire ce qui les définit en prima facie comme facteurs de risque pour la sécurité de l'Union, soit parce qu'ils sont idéologiquement hostiles, soit, et c'est bien plus neuf, parce que leurs citoyens sont à titre ou à un autre des individus indésirables dans l'espace de l'Union et que l'on considère qu'ils cherchent à venir sur le territoire, non à des fins de tourisme ou parce qu'ils fuient les persécutions, mais parce qu'ils cherchent à séjourner illégalement dans l'Union.

Dans un second temps, la décision individuelle sur chaque demande peut revenir sur le principe de l'exclusion et la lever, dans le cas individuel. Dans ce dispositif, la politique d'accueil est dérogatoire à la politique du soupçon. Et c'est d'ailleurs ce que les fonctionnaires en charge comprennent bien lorsqu'on les interroge sur les intentions des gouvernants, même lorsqu'ils sont perdus devant les enjeux techniques qui souvent masquent les conflits de compétence entre la Commission et les Etats. Néanmoins, historiquement, on peut considérer que cette ouverture à de nombreux individus venant de pays dont on se méfie est plutôt positive car elle permet malgré tout à nombre d'entre eux de voyager vers l'Europe de Schengen ${ }^{7}$, du moins si leurs Etats les laissent partir et ne créent pas des délits d'émigration ${ }^{8}$.

Le premier temps, celui de l'établissement de la liste des pays, relève depuis peu de la Commission qui cherche donc à harmoniser les positions des Etats entre eux en faisant disparaître les listes additionnelles que certains Etats avaient créées. En revanche, la Commission estime que l' octroi et le refus des visas à titre individuel relève des seuls Etats $^{9}$. Cela laisse les individus avec très peu de recours devant le refus d'un consulat particulier comme le montre Claire Saas. Paradoxalement le système de justification du rôle spécifique de la Commission est inversé puisque c'est elle qui met l'accent sur la dangerosité des flux d'individus et bien moins sur les politiques gouvernementales des Etats tiers. Il en résulte des chassés-croisés où chacun renvoie la responsabilité de l'inefficacité globale du système sur l' autre partenaire.

La dichotomie entre le caractère diplomatique des relations interétatiques et le caractère illégal des activités de l'individu mise en avant par la Commission masque en fait une vision en termes de « menaces transverses à la sécurité intérieure " provenant de certaines communautés des pays tiers. Ces termes de menaces à la sécurité de l'Union qui ne sont pas dans le texte sont revenus en permanence dans les entretiens. 
Le critère implicite des menaces transverses relie en fait les trois critères explicites de la Commission. Il se traduit par la défiance à l'égard des flux transnationaux de population, en particulier tous ceux qui bougent beaucoup (diasporas et réfugiés) ou ceux qui sont prosélytes (musulmans). Ces individus appartenant à des groupes à profil particulier affecteraient la sécurité intérieure de l'Union en servant de soutien potentiel aux terroristes ou en participant à des activités criminelles. La construction de la défiance passe alors certes par l'attitude des gouvernements les uns envers les autres, par leur appartenance à la liste des rogue states ou des pays narco-trafiquants mais aussi par le fait qu'ils sont pauvres, ou en conflits et que leurs populations peuvent avoir des raisons de fuir les conditions de vie qui leur sont faites. Mais, outre les attitudes des gouvernements, ce qui préoccupe la Commission dans l'élaboration de la liste c'est que ces flux de population peuvent être considérés comme dangereux ; soit parce qu'ils généreraient du terrorisme, du radicalisme religieux, de la criminalité, soit parce qu'ils appartiendraient à des catégories de candidats à la migration dont l'Union européenne ne veut pas, soit encore parce que la situation politique est telle qu'il leur faut fuir leur pays et chercher asile.

On retrouve ici dans l'établissement de la liste et dans la présentation orale qui en est faite dans les entretiens, la construction d'un continuum d 'insécurités qui sont reliées les unes aux autres et qui englobent au-delà des acteurs gouvernementaux les pratiques sociétales de certains groupes sociaux de ces pays ${ }^{10}$. La considération sur l'illégalité de la migration individuelle masque difficilement une conception qui joue sur des "flux " et non des individus et une conception qui assimile pauvreté, criminalité, illégalité, migration régulière ou non et demandeurs d'asile.

La liste des pays dont les ressortissants sont soumis à visa est donc éminemment discutable, bien qu'elle n'ait pas été discutée. Ses critères qui mêlent migration et criminalité ou idéologie du régime dont viennent les individus ne sont pas cohérents. La réponse dans les entretiens à cette objection est de minimiser ce point. Il y aurait plus ou moins de souplesse selon les Etats. Intransigeance envers les pays terroristes, souplesse avec les pays d'où viennent les demandeurs d'asile. Mais on peut en douter. N'y a-t-il pas, pour eux aussi, une forte intransigeance mais plus masquée parce que plus illégitime? La question est alors : quelle est au sein de la liste des pays soumis à visa, la hiérarchie des pays les plus «surveillés »? N'y a-t-il pas un classement autre qu'alphabétique des pays et qui établit une gradation des menaces? Et si oui, qui produit ce classement et est-il appliqué uniformément? Echange-t-on des peurs à propos des risques en les "communautarisant» ou chacun établit-il ses priorités en étant finalement peu intéressé à la gestion des peurs des autres? Selon nous, et bien que cela soit nié officiellement, l'annexe $5 b$ - qui est confidentielle - sert à établir cette hiérarchie en créant une liste "extrême noire" des pays qui sont, non seulement soumis à visa, mais si dangereux que l'on ne fait pas confiance aux autres autorités centrales pour leur accorder un visa ${ }^{11}$.

C'est alors la vision de la dangerosité des individus qui joue - criminalité et migration inclues - mais justifiée par une certaine géopolitique - ou idéologie - qui relie islam, terrorisme et migration (et qui a été renforcée depuis le 11 septembre 2001). C'est aussi une peur plus globale de déstabilisation des identités nationales - au nom du danger de flux massifs en provenance de l'étranger qu'ils soient légaux ou non, migratoires ou de réfugiés qui affecteraient les équilibres des pays receveurs de ces flux- dont on connaît le lien avec les idéologies d'extrême droite. Ces menaces ne relèvent en effet guère de "faits ». Elles sont des peurs construites autour de quelques phénomènes de violence 
mais aussi autour de simples droits qu'il faudrait pourtant accorder aussi aux individus des pays du tiers monde au lieu de les immobiliser chez eux. Elles sont par ailleurs hiérarchisées selon des considérations variables venant de débats transatlantiques, européens, nationaux, ou bureaucratiques dont le SIS est le reflet ${ }^{12}$. Chaque pays a sa propre gestion et sa propre amplitude de peur. Il les échange ou non avec les autres pays. Il est rare qu'il se préoccupe à la frontière des peurs des autres pays concernant des individus non inscrits dans le SIS. Néanmoins certaines catégories de peur se communautarisent plus vite que d'autres - par exemple celles transfrontières sur les gens du voyage qui devient très rapidement une suspicion sur les Roumains en général. $C^{\prime}$ 'est à ce niveau sans doute qu'il existe un lien entre le SIS et Europol au sens où les équipes d' analyse d'Europol visent à construire les catégories qui seront ensuite appliquées par les Etats pour rentrer leurs données Schengen sur les « indésirables » et qu'elles déterminent aussi les visions des agents consulaires.

Ainsi, si les trois critères sont utilisés par la Commission, c'est que le seul critère des politiques étrangères des Etats remettrait en cause sa prétention à gérer les mécanismes d'entrée des individus des pays à risque. Il lui faut s'appuyer sur une logique différente plus centrée sur les individus pris en tant que flux de population. Mais, qui est finalement responsable en cas de refus de visa à une catégorie de personnes? Les autorités consulaires de l'Etat où elles ont été demandées ou les autorités qui ont mis en place les critères? La Commission peut-elle échapper aux critiques alors qu'elle établit les critères qui seront ensuite appliqués ? Pour répondre partiellement à ces questions, il faut maintenant considérer si les conditions d'octroi et refus d'un visa sont soumises à harmonisation ou non.

Il est donc nécessaire de différencier les Etats des individus qui en sont les ressortissants. Ce sont les ressortissants de tel ou tel Etat qui sont considérés comme menaçants ou dangereux. Cette menace ou ce danger, incarné par les individus, rejaillit le plus souvent sur l'Etat qui sera inscrit sur la liste noire pour cette raison. Celle-ci n'est plus limitée aux rogue states, elle peut prendre la tournure inflationniste qu'on lui connaît.

Les Visas Schengen, les instructions consulaires communes, la méfiance des Etats entre eux et le rôle de la Commission

La clé du système de contrôle des frontières Schengen se trouve ainsi non pas dans la systématicité de la vérification des documents aux frontières, mais dans les modalités de profilage et d'identification des menaces venant de l'étranger. La première étape pour identifier ces menaces et risques est, nous l'avons vu, le profilage par nationalité avec l'imposition de visas obligatoires sur tous les ressortissants des pays à risque. La deuxième étape est d'identifier les individus qui ne constituent pas une menace parmi les individus de nationalité douteuse aux yeux de l'Union et de s'assurer que seulement ces personnes vont se voir octroyer un visa. C'est le travail à la fois des ministères nationaux des Affaires étrangères qui donnent les règles et des autorités diplomatiques et consulaires sur place ${ }^{13}$.

Les instructions consulaires communes : instruction aux agents à l'égard des demandeurs

Les instructions consulaires, dans le souci d'homogénéisation des critères et des pratiques a des phrases qui, dirigées vers les agents consulaires, ont le mérite de la clarté.

"Il est rappelé que les préoccupations essentielles qui doivent guider l'instruction des demandes de visa sont: la sécurité des Parties contractantes et la lutte contre l'immigration clandestine 
ainsi que d' autres aspects relevant des relations internationales. Selon les pays, l'une pourra prévaloir sur les autres mais aucune ne devra jamais être perdue de vue.

S'agissant de la sécurité, il convient de s'assurer que les contrôles nécessaires ont été effectués : consultation des fichiers des non-admis (signalements aux fins de nonadmission via le Système d'Information Schengen, consultation des autorités centrales pour les pays soumis à cette procédure).

S'agissant du risque migratoire, l'appréciation relève de l'entière responsabilité de la Représentation diplomatique ou consulaire $»^{14}$. "La coopération consulaire sur place, plus généralement, portera sur l'évaluation des risques migratoires. Elle aura pour objet notamment la détermination de critères communs pour l'instruction des dossiers, l'échange d'informations sur l'utilisation de faux documents, sur les éventuelles filières d'immigration clandestine et sur les refus de visa dans le cas de demandes manifestement non fondées ou frauduleuses. Elle devra également permettre l'échange d'informations sur les demandeursbona fideainsi que la mise au point, en commun, de l'information du public sur les conditions de la demande du visa Schengen " ${ }^{15}$.Ainsi, «l'examen des demandes de visa vise à détecter les candidats à l'immigration qui cherchent à pénétrer et à s'établir dans le territoire des Parties contractantes, sous le couvert de visa pour tourisme, études, affaires, visite familiale. Il convient à cet effet d'exercer une vigilance particulière sur les "populations à risque», chômeurs, personnes démunies de ressources stables etc. En cas de doute portant notamment sur l'authenticité des documents et la réalité des justifications présentées, la Représentation diplomatique ou consulaire s'abstiendra de délivrer le visa ».

Des trois critères, le critère du niveau de vie pour l'octroi du visa est semble-t-il le plus fréquent dans la prise de décision car le plus commun. Mais c'est aussi le plus illégitime car il transforme le refus de visa qui peut se comprendre à l'égard du crime et de certains motifs de sécurité nationale en une arme dissuasive contre le «désir migratoire ». On suppose que ceux qui sont assez riches pour voyager reviendront chez eux alors que l'on en doute pour les plus pauvres. Ceux-ci sont censés vouloir tricher pour profiter du Welfareoccidental. La première qualité du voyageur est-elle de pouvoir être économiquement autonome et bon consommateur? Il le semble. Chaque personne soumise à l'obligation de visa est alors considérée comme un risque d'immigration clandestine. L'individu arrive comme suspect. Son désir de voyager est interprété comme un désir de s'établir. Il devient par définition un risque s'il est pauvre, chômeur ou démunie de ressources stables, ce qui disqualifie très vite les étudiants, les artistes et certaines professions libérales. Sous le couvert du terme "population à risque ", on transforme les populations pauvres ou « instables » en populations dangereuses.

Les instructions consulaires communes se réfèrent à ces différences entre riches et pauvres, via le choix des agences de voyages: "Cette obligation de l'entretien personnel peut être supprimée au cas où un organe de bonne réputation et digne de confiance serait dans la possibilité de se porter garant de la bonne foi des individus concernés ». Phrase lourde de sens et qui débouche sur des risques non négligeables de corruption des agents consulaires par ces agences de voyage. La Bulgarie en a été un exemple. Il a certes été indiqué dans les entretiens que ces pratiques étaient problématiques et assez rares, mais cela renforce l'idée qu'il existe des informations selon lesquelles le choix de la compagnie aérienne est pris en compte comme étant un indicateur de bona fide. Dès lors, si l' individu a acheté un billet avec le transporteur national du pays où il se rend ou l'une des principales compagnies de son pays, sa bona fide est renforcée. Que les avantages commerciaux soient reliés à l'obtention de visa est sans doute problématique 
en termes de légitimité. Même sans supposer des collusions entre les compagnies aériennes, les agences de voyage et les décisions des consulats, il pourrait résulter que l'obtention ou non du visa résulte du choix malheureux d'un consommateur modeste quant à l'agence de voyage ou la compagnie aérienne. Les instructions consulaires ont un statut ambigu: entre texte normatif et manuel donnant des recettes aux agents consulaires, elles oscillent entre un discours qui se veut reflet de la politique de l'Union sur le plan officiel et un discours qui en donne la grammaire effective, celui de la mise à l'écart des étrangers pauvres.

Un «texte» implicite autour de l'immigration invasion - qui oublie totalement les effets pendulaires des migrations effectives ainsi que le fait que le désir de voyager est un des critères les plus marquants de la fin de siècle - structure l'ensemble des représentations et des actions que l'on demande aux agents consulaires de faire. La coopération consulaire risque dès lors de renforcer les suspicions des fonctionnaires contre l' individu qui est par définition d'autant plus une menace migratoire, qu'il est pauvre et villageois, inconnu des autorités consulaires locales... alors que quelqu'un de « recommandé ", habitant la ville, passant par une agence de voyage sera plus favorisé. Une forme de clientélisme ne se met-elle pas alors en place en étant encouragée officiellement au nom de la prévention du risque migratoire?

On voit la dérive possible inscrite dans le texte même lorsque l'on évoque la vérification d'autres documents en fonction de la demande et que l'on écrit « Le nombre et la nature des justificatifs dépendent du risque éventuel d'immigration illégale et de la situation locale (par exemple, monnaie transférable ou non) $»^{16}$. Si l'on peut exiger plus de documents pour certaines personnes que pour $d$ ' autres en fonction de leur richesse ou de toute autre appréciation du risque migratoire, ne favorise-t-on pas à l'intérieur même d'un texte publié au Journal Officiel des Communautés européennes, l'émergence de critères « ethniques » ou " racistes » qui n'ont pas à s'exprimer comme tels? Cette enquête préliminaire ne peut trancher, mais il faudrait une étude approfondie sur le danger de l'émergence de formes de racisme institutionnalisé dans les instructions consulaires elles-mêmes.

On semble alors se diriger vers une société globale de sédentaires, de touristes et de vagabonds où seules les élites économiques pourront finalement voyager, les autres étant piégées dans le local comme le signale Zygmunt Bauman dans le " coût humain de la globalisation $»^{17}$. Néanmoins, être riche ne suffit pas non plus. Zygmunt Bauman, dans son livre magnifique, néglige ce point. Les riches sont contrôlés aussi lorsqu'ils circulent et depuis le 11 septembre 2001 c'est même sur eux que l'on expérimente les techniques de contrôle les plus intrusives (biométrie, couloir d'accès spécifiques, enquêtes préalables) en leur vendant ces techniques au nom de leur meilleure protection ${ }^{18}$.

Des refus de visa leur sont opposés dans certains pays. Mais dans ce cas, on voit le critère des croyances religieuses être appliqué implicitement. Alors qu'il n'est pas un critère déclaré, il semble que le port ostensible de certains symboles de l'islam militant soit retenu contre les demandeurs, même riches.

La variabilité des critères selon les contextes locaux

L'instruction consulaire évoque le fait que les critères appliqués peuvent varier d'un pays à l'autre et cela semble logique, mais peuvent-ils concerner une minorité particulière ou une zone géographique ou un genre particulier $?^{19}$ Au-delà de ces instructions, comme nous le verrons plus loin, les entretiens font aussi état de « grilles 
géopolitiques » qui circuleraient et qui reposeraient sur des traits ethniques, sur des croyances religieuses ou idéologiques de tel ou tel groupe affecté de commentaires sur leur dangerosité, et parfois de noms d'individus qui en seraient des "leaders» et qui seraient dès lors encore plus dangereux. On peut s' interroger sur ces pratiques encouragées par les textes. Est-il raisonnable de laisser circuler entre les consulats des listes de noms d'individus à surveiller, des e-mails entre agents consulaires qui, de la sorte, ne constituent pas un "fichier", des analyses géoculturelles dont la lecture ressemble plus aux tropismes du colonialisme le plus ancien qu'à des analyses ethnologiques ou sociologiques? Les instructions consulaires communes ne prévoient aucun contrôle indépendant à l'égard des informations circulant entre les autorités consulaire. Pourquoi un tel «trou » dans la législation? Comment peut-on tolérer et même encourager officiellement la constitution d'un troisième système d'information sur les demandeurs de visas en plus du SIS et du système national reliant les autorités consulaires ? En effet, en ce qui concerne l'appréciation des justificatifs donnée par « les Représentations diplomatiques et consulaires des Parties contractantes, ces dernières peuvent convenir de modalités pratiques adaptées aux circonstances locales $»^{20}$.

Dès lors les instructions consulaires communes justifient à l'avance les disparités qui éclatent d'un consulat à l'autre, d'un pays à l'autre en renvoyant la responsabilité des dysfonctionnements sur l'absence de coopération d'un réseau qui devrait s'instituer entre les consulats et elles encouragent la mise en place d'un fichier qui ne dit pas son nom pour ne pas permettre un accès aux données de ce fichier. Or, comment appeler des listes de noms échangés, via e-mails de manière informelle, sinon un fichier masqué ? ${ }^{21}$

Là encore les instructions consulaires évoquent ce fichier sans le nommer : «A l'inverse, les contrôles seront allégés pour les demandeurs reconnus comme étant des personnesbona fide, ces informations étant échangées dans le cadre de la coopération consulaire ". Ainsi, théoriquement, ces listes seraient des listes blanches favorisant certaines personnes mais plusieurs entretiens ont fait état de listes noires ou de listes doubles (blanches et noires) suivant l'interprétation du passage suivant : «En vue de l'appréciation de la 'bonne foi' du demandeur, les Représentations vérifient si le demandeur fait partie des personnes de 'bonne foi' reconnues comme telles dans le cadre de la coopération consulaire sur place ».

En laissant faire, voire en encourageant la mise en place de telles mesures sans contrepoids en termes de libertés, le texte de l'instruction consulaire commune n'est pas un texte protecteur des droits des individus. Il recommande explicitement des attitudes qui ne seraient pas tolérées si nos ressortissants en souffraient. Plus grave encore, les pratiques vont au-delà de ce texte.

\section{NOTES}

1.. Liste commune des pays tiers dont les ressortissants sont exemptés de l'obligation de visa. 
2. . Liste commune des pays tiers dont les ressortissants sont soumis à l'obligation de visa.

3. . L'appréciation de la notion de l'ordre public peut varier très sensiblement d'un Etat-membre à un autre. Dans l' affaire Donatella Calfa (aff. C-386/96 du 19 janvier 1999), la Cour de Justice des Communautés Européennes a été confrontée à cette difficulté. Il s'agissait d'une ressortissante italienne condamnée en Grèce pour détention de stupéfiants à usage personnel à une peine de trois mois d'emprisonnement et à une mesure d'expulsion emportant une interdiction définitive de revenir sur le territoire grec. La question de la compatibilité d'une mesure obligatoire d'expulsion assortie d'une interdiction du territoire définitive avec le principe de libre circulation et de citoyenneté européenne était donc posée. La Cour a tout d'abord rappelé que les Etats membres pouvaient prendre des mesures d'éloignement à l'égard de ressortissants communautaires, lorsque des raisons liées à l'ordre public les justifient. Les arrêts Bouchereau et Van Duyn avaient posé le principe selon lequel « le recours à la notion d'ordre public suppose, en tout cas, l' existence, en dehors du trouble pour l'ordre social que constitue toute infraction à la loi, d'une menace réelle et suffisamment grave, affectant un intérêt fondamental de la société, menace qui ne peut par contre pas être déduite, en soi, de la simple existence d'une décision de condamnation ». Selon la Cour, l'expulsion imposée par la législation grecque ne résulte pas d'une appréciation spécifique du comportement du coupable mais découle d'une procédure quasi-automatique. Cette disposition pénale grecque est donc contraire au droit communautaire, du fait de son automaticité. Si la procédure automatique est sanctionnée, le fait de considérer que la détention de drogues douces à usage personnel est pénalement répréhensible $n$ 'est absolument pas discuté par la Cour de Justice. C'est pourtant le point crucial : en Grèce, ce comportement est pénalement répréhensible. Dans d' autres pays de l'Union, la détention de drogues douces a été dépénalisée, de jure en Belgique et aux Pays-Bas, et de facto en Allemagne. La Cour reconnaît donc une certaine «marge d'appréciation » des Etats pour déterminer ce qui appartient à l'ordre public, ce qui ne facilite pas la clarté d'une telle notion.

4. . En faisant appel à ce troisième critère des relations internationales, l'Union européenne adopte une position doublement extensive quant à la détermination des personnes dont elle ne souhaite pas la présence sur son territoire. Dans un premier temps, chaque Etat membre devra se montrer solidaire de la position d'un autre Etat membre et considérer qu'un pays qui, pour lui, ne revêt pas les caractéristiques nécessaires pour soumettre ses ressortissants à visa, figure sur la liste noire. On a choisi le plus grand dénominateur commun. Dans un second temps, cette décision ne sera pas prise indépendamment des autres pays situés dans le même espace régional, sur lesquels rejaillira la soumission à visa : l'Algérie ne sera pas considérée indépendamment de sa situation géographique, mais par rapport à l'espace du Maghreb. Cela ressemble bien plus à un réseau - les Etats de l'Union entre eux et leur relation avec diverses zones géographiques - qu'à une harmonisation.

5. . Les Roumains le savaient bien quand ils étaient soumis au régime de visa. Les Macédoniens en font toujours l' expérience.

6. On sent une gêne lorsque l'on demande des précisions sur les risques afférant à ces trois critères et sur les liens entre eux. La liste, lorsqu'elle est citée de mémoire, est par trop celle des pays islamiques, des pays pauvres et celle des pays en conflits où les demandeurs d'asile sont importants. La stratégie de citer la liste des pays exempts de 
visas étant, elle, par trop celle des pays riches occidentaux. En analysant dans la prochaine partie l'annexe $5 b$, nous ferons une analyse plus détaillée des « discours sur la liste des pays soumis à visa » en signalant quelles sont les saillances et les raisons du choix de ces pays sensibles parmi les pays sensibles.

7.. Voir les statistiques sur les taux d'acceptation et de refus (Cultures \& Conflits, $n^{\circ} 50$, Eté 2003).

8. . La Tunisie est en train de s'engager dans cette voie et plusieurs pays africains seraient sous pression des Etats de l'Union pour créer ce type de délit. Communication de Salvatore Palidda à la réunion ELISE, CEPS, Mars 2003.

9. . Entretien avec Mr Fortescue directeur général de la DG JAI à l'époque de l' entretien (mars 2001). Composition actuelle de la DG JAI, voir annexes (Cultures \& Conflits, $n^{\circ} 50$, Eté 2003).

10. . Sur cette notion de continuum d'(in)sécurité, voir Didier Bigo « Polices en réseaux », op. cit.

11. Voir supra.

12. . Sur la notion de construction des menaces, voir le numéro 31-32 de Cultures \& Conflits, Sécurité et immigration, en particulier les articles de Jef Huysmans, Didier Bigo et Ayse Ceyhan.

13. . Par commodité nous emploierons la formule autorité ou agent consulaire.

14. . JOCE C313, p. 11.

15. . JOCE C313, p. 21.

16. . ICC point 1.4, JOCE C313, p. 12.

17. . Bauman Zygmunt, op. cit.

18. . Le 11 septembre n'a fait que renforcer une tendance de technologies déjà existantes en en justifiant le coût et la généralisation. Voir conclusion de cet article.

19. . C'est ce que laissent supposer des entretiens à propos de la liste de l'annexe $5 b$. Le fait que cette annexe soit gardée confidentielle est aussi la preuve de la gêne des autorités à cet égard.

20. . ICC 1.4 JOCE C313, p.12.

21. . Voir supra.

INDEX

Mots-clés : police à distance, Visa

Index géographique : Schengen

Thèmes : Schengen (convention) 